
\title{
Besearch Sfunare \\ Clinical Characteristics and Nomogram for Adult Medullary Thyroid Carcinoma
}

\section{Xiao Li}

Linyi people's Hospital

Yu-jia Lin

Jilin Cancer Hospital

Xian-zhi Wang

Linyi people's Hospital

Xiang-min Zeng

Weifang Yidu Central Hospital

Qiang li

Jilin Cancer Hospital

Xu-he Zhang ( $\nabla$ zhangxuhe1848@163.com )

Department of head and neck surgery, Jilin Cancer Hospital, Changchun, Jilin, China.

\section{Research Article}

Keywords: Medullary thyroid carcinoma, SEER, treatment and prognostic factors, nomogram

Posted Date: January 4th, 2021

DOl: https://doi.org/10.21203/rs.3.rs-136254/v1

License: (c) (i) This work is licensed under a Creative Commons Attribution 4.0 International License.

Read Full License 


\section{Abstract}

Objectives: The present study aimed to investigate the clinical characteristics and independent prognostic factors in adult patients with medullary thyroid carcinoma (MTC), followed by construction of nomogram-based prognostic prediction model for adult MTC.

Methods: Relevant subjects were retrieved from Surveillance, Epidemiology, and End Results (SEER) program from January 1, 2004 through December 31, 2014. According to the multivariate analysis, the 3and 5-year cancer-specific survival (CSS) in MTC patients was predicted by constructing a nomogram.

Results: In total, 1279 patients diagnosed with primary MTC were extracted from the SEER database. The 3-, 5-and 10-year CSS rates were $91.11 \%, 88.32 \%$, and $81.63 \%$, respectively. The Cox proportional hazards model revealed that advanced AJCC stage and elderly age were independent unfavorable prognostic factors, and that surgery and radical lymph node dissection were protective prognostic factors (all $P]$ 0.05). In addition, radiotherapy was independently related to the decreased survival. The C-index of nomogram was 0.902 (95\% Cl: 0.878-0.926) for the internal validations. Calibration plot revealed satisfactory agreement between the actual findings and predicted outcomes.

Conclusion: The nomogram could individually and precisely predict the CSS of MTC patients, which could be used to facilitate in individualized prognostic assessment evaluation and clinical decisionmaking.

\section{Introduction}

Medullary thyroid carcinoma (MTC), an uncommon cancer, occupies about $10 \%$ of thyroid cancers and $14 \%$ of the thyroid cancer-related deaths ${ }^{1}$. MTC has a low morbidity rate; therefore, relative to other common thyroid cancer subtypes, the pathological characteristics and prognostic outcome of MTC are rarely known by most clinicians, even for the experienced ones. In this regard, it is urgently needed to pay more attention to $\mathrm{MTC}^{2}$. Considering that MTC shows diverse clinicopathological characteristics and prognostic outcome in children compared with adults, it is greatly significant to separately explore adult patients ${ }^{3}$.

Due to the relatively low incidence of MTC, studies concerning the patient characteristics, therapeutic regimen and prognosis of MTC are rarely available, especially for adults ${ }^{4}$.Nevertheless, the prognosis of MTC is affected by multiple factors, that is, the outcome of MTC cannot be accurately predicted by any single factor. To this end, herein, a prognostic model was constructed by incorporating several prognostic factors based on large samples, aiming at the individualized prediction of survival in adult MTC patients ${ }^{5,6}$.

Nomogram, a statistic-based tool by incorporating multiple independent prognostic factors, have been extensively adopted for precise survival prediction for cancer patients, including bladder cancer, gastric cancer, lung cancer and breast cancer ${ }^{7-11}$. The Surveillance, Epidemiology, and End Results (SEER) 
database provides adequate clinical data on MTC patients, rendering prognostic evaluation for MTC. In a previous study, the prognosis nomogram of medullary thyroid cancer has been established, but it was not specifically for adults, and the factors of radiotherapy and chemotherapy were not considered ${ }^{12}$. In the present study, relevant data were extracted from the SEER database for the identification of independent prognostic factors that influenced the cancer-specific survival (CSS) of adults MTC patients, followed by construction of a nomogram to predict CSS rate in adults MTC patients.

\section{Methods}

\section{Ethics statement}

Starting in 1973, the SEER program formulated by the National Cancer Institute (NCI)is the populationbased database developed to develop the all-sided sources, which involves about $30 \%$ US populations across diverse geographic regions and is updated every year ${ }^{13}$. The SEER Research Data Agreement (No.11357-Nov2018) was signed for obtaining the related data from this database, and data were additionally searched in accordance with corresponding guidelines. All the collected information was public and de-identified. The ethical approval was waived by the institutional review board since the Office for Human Research Protection regarded data analysis as non-human subjects.

\section{Study population}

The eligible cases were screened using the SEER*State v8.3.5 approach (released on March 6th, 2018). In the present work, we included 18 SEER regions between 2004 and 2014. Patients conforming to the following criteria were enrolled: (1) those with primary MTC with the age of more than 18 years; (2) those with MTC diagnosed according to the third version of the International Classification of Disease for Oncology (ICD-0-3; coded as 8510/3 and 8345/3). At the same time, patients conforming to any one of the following conditions were excluded from this study: (1) patients with more than one primary tumor;(2) those who were diagnosed at the age $\mathbb{1 8}$ years; (3) those with clinical diagnosis, or those diagnosed based on autopsy or the death certificate; (4) those with insufficient clinicopathological data, like age at diagnosis, race, martial status, surgery mode and AJCC stage; (5) those with the survival time of less than 3 months postoperatively; and (6) those with no data on prognosis. The rest participants were included into the initial SEER cohort.

\section{Covariates and endpoint}

Altogether 12 clinicopathological features shown below were examined, including gender (female, male); age ( $<40,40-65,>65$ years); race (black, white or others); marital status(married, unmarried); insurance status (uninsured/unknown, any medicaid/insured); tumor size $(₫ 2,2-4$, and $₫ 4 \mathrm{~cm})$; solitary/multifocal (solitary tumor, multifocal tumor, unknown); lymph node dissections (LND, none or biopsy, 1-3 or $\geq 4$ regional lymph nodes retrieved), AJCC stage (I, II, III, and IV), surgery mode (non-surgery, lobectomy, subtotal/total thyroidectomy), chemotherapy (yes, no/unknown) and radiotherapy(yes, no/unknown). To be specific, the divorced, or separated or single (having domestic partners or never married) or widowed 
were assigned as unmarried. For analyzing the association between survival and age, we deemed age (< $40,41-65,>65$ years)as the categorical variable. Meanwhile, race was classified as black, white, or other. Besides, the qualified patients were further grouped according to the AJCC TNM 8th classification system.

Cancer-specific survival (CSS) was considered as the primary endpoint of the research, which was defined as the duration from diagnosis to MTC-caused death. We preliminarily determined the deadline till November 2018 based on the SEER 2018 submission database. Finally, the deadline was set as November 31st, 2018.

\section{Statistical analysis}

The Kaplan-Meier (KM) method was used to plot cumulative survival curves, which were then compared through the log-rank test. Moreover, the significant prognostic factors were identified by univariate as well as multivariate Cox proportional hazards regression analysis, and presented with hazard ratios (HRs) as well as the corresponding $95 \%$ confidence intervals (Cls). In the meantime, variables of $P<0.1$ identified by univariate analysis were introduced to multivariate analysis. Then, the $\mathrm{R}$ software rms package version 3.5.1 (http://www.r-project.org/) was utilized to establish nomograms to predict the CSS at 3 and 5 years in accordance with multivariate analysis. Besides, the Concordance index (C-index) was used to evaluate the nomogram performance, with the value ranging from 0.5 (poorest model) to 1.0 (optimal model). Typically, nomograms that had the C-index of 0.7 were indicative of excellent predicting ability ${ }^{14}$. In line with the bootstrap 1000 resampling validation, we used the calibration curves to assess the consistency of nomogram-predicted survival with the real survival. At last, the ROC curves were plotted and the area under the curve (AUC) values were calculated to evaluate the accuracy in predicting the 3-and 5-year prognosis by the use of $\mathrm{R}$ software survival ROC package. A difference of $P<0.05$ (two-sided) was deemed to be statistically significant.

\section{Results}

Clinicopathological characteristics of patients.

Altogether 2105 MTC cases were enrolled into the SEER database from 2004 to 2014. In line with the above-mentioned patient selection criteria, a total of 1279 qualified cases were recruited into the present work. Figure 1 displays the flow chart of patient screening. Table 1 presents the basic patient characteristics. Specifically, a majority of our cases (83.50\%) were white, meanwhile, $65.52 \%$ were married and $58.95 \%$ were female. The age at diagnosis ranged from 20 to 99 years, with the median of 53 years, and most patients (58.41\%) were in the 40-65 age group. The average tumor size was $2.3 \mathrm{~cm}$. Over $1 / 2$ cases $(53.32 \%$ ) were at AJCC stage I and II, and $10.09 \%$ cases had extended lesion to the adjacent normal tissues or lymph node metastasis (LNM). 
Table 1

Demographic, Clinical, and Pathologic Characteristics of Patients with Histologically Confirmed Medullary

Thyroid Cancer, SEER 2004-2014 ( $n=1279)$

\begin{tabular}{|c|c|c|}
\hline Characteristics & Number & Percent \\
\hline \multicolumn{3}{|l|}{ Insured status } \\
\hline uninsured/unknown & 339 & $26.51 \%$ \\
\hline any medicaid & 98 & $7.66 \%$ \\
\hline insured & 842 & $65.83 \%$ \\
\hline \multicolumn{3}{|l|}{ Age } \\
\hline$<40$ & 247 & $19.31 \%$ \\
\hline $40-65$ & 747 & $58.41 \%$ \\
\hline$₫ 65$ & 285 & $22.28 \%$ \\
\hline \multicolumn{3}{|l|}{ Gender } \\
\hline male & 525 & $41.05 \%$ \\
\hline female & 754 & $58.95 \%$ \\
\hline \multicolumn{3}{|l|}{ Marital status } \\
\hline unmarried & 441 & $34.48 \%$ \\
\hline married & 838 & $65.52 \%$ \\
\hline \multicolumn{3}{|l|}{ Race } \\
\hline white & 1068 & $83.50 \%$ \\
\hline black & 113 & $8.84 \%$ \\
\hline other & 98 & $7.66 \%$ \\
\hline \multicolumn{3}{|l|}{ AJCC stage } \\
\hline stage I & 409 & $31.98 \%$ \\
\hline stage II & 273 & $21.34 \%$ \\
\hline stage III & 129 & $10.09 \%$ \\
\hline stage IV & 468 & $36.59 \%$ \\
\hline \multicolumn{3}{|l|}{ Tumor size } \\
\hline$\varangle 2 \mathrm{~cm}$ & 602 & $47.07 \%$ \\
\hline $2-4 \mathrm{~cm}$ & 442 & $34.56 \%$ \\
\hline
\end{tabular}




\begin{tabular}{|lll|}
\hline Characteristics & Number & Percent \\
\hline $\mathbb{} 4 \mathrm{~cm}$ & 235 & $18.37 \%$ \\
\hline Solitary/multifocal & & \\
\hline solitary tumor & 881 & $68.88 \%$ \\
\hline multifocal tumor & 341 & $26.66 \%$ \\
\hline unknown & 57 & $4.46 \%$ \\
\hline Surgery & & \\
\hline no surgery & 101 & $7.90 \%$ \\
\hline lobectomy & 88 & $6.88 \%$ \\
\hline total/subtotal thyroidectomy & 1090 & $85.22 \%$ \\
\hline Lymph node dissection & & \\
\hline none or biopsy & 392 & $30.65 \%$ \\
\hline 1 to 3 regional lymph nodes & 164 & $12.82 \%$ \\
\hline$\geq 4$ regional lymph nodes & 723 & $56.53 \%$ \\
\hline Chemotherapy & & \\
\hline no/unknown & 1193 & $93.28 \%$ \\
\hline yes & 86 & $6.72 \%$ \\
\hline Radiotherapy & 1093 & $85.46 \%$ \\
\hline no/unknown & $186.54 \%$ \\
\hline yes & & \\
\hline
\end{tabular}

The type of surgery was recorded in 1178 patients (92.10\%). Among all the patients undergoing surgery, $22 \%$ of them received total/subtotal thyroidectomy without lymph node dissection; $14 \%$ received limited lymph node dissection and $64 \%$ underwent modified/radical lymph node dissection. In addition, $6.88 \%$ of patients received lobectomy, while $7.90 \%$ of patients did not undergo operation. Adjuvant radiotherapy was performed on $14.54 \%$ of patients, including external beam radiation $(12.67 \%)$, radioisotopes $(1.72 \%)$ and radioactive implants $(0.15 \%)$. The majority of patients $(88.71 \%)$ receiving adjuvant radiotherapy had an advanced stage (stage III and IV). In addition, the number of patients receiving adjuvant chemotherapy was less (6.72\%), who were generally burdened with advanced stage.

\section{Survival and Prognostic factors}


Patients were followed up for 0-131 (median, 47) months. The CSS rates at 3, 5 and 10 years, respectively were $91.11 \%, 88.32 \%$ and $81.63 \%$. Figure 2 shows the CSS survival curve. According to univariate analysis, gender $(P=0.001)$, age $(P<0.001)$, tumor size $(P<0.001)$, AJCC stage $(P<0.001)$, LND $(P<$ $0.001)$, surgery $(P<0.001)$, radiotherapy $(P<0.001)$ and chemotherapy $(P<0.001)$ were identified as the CSS predicting factors (Fig. 3). Besides, upon multivariate analysis, the older age [( $₫ 65$ years)HR:2.971, 95\%Cl: $1.577-5.497, P<0.001]$ and the advanced AJCC stage [(stage II)HR5.693, 95\%Cl: 1.205-26.909, $P$ $=0.028$; (stage III)HR:10.474, 95\%Cl: 1.978-55.472, $P=0.006$; (stage IV)HR:42.797, 95\%Cl: $10.006-$ 183.040, $P<0.001]$ were identified as the independent prognostic factors to predict the dismal prognosis; while surgery[(lobectomy) HR:0.286, 95\% Cl: 0.107-0.764,P=0.013;(subtotal/total thyroidectomy) HR:0.244, 95\%Cl: $0.125-0.476, P<0.001]$ and radical LND [( $\geq 4$ regional lymph nodes) HR:0.652, 95\%Cl: $0.348-1.221, P=0.039]$ were identified as the factors to predict positive prognosis. Besides, radiotherapy was associated with the unfavorable survival (HR: $1.820,95 \% \mathrm{Cl}: 1.270-2.608, P=0.001)$ (Table 2). 
Table 2

Univariate and multivariate Cox proportional hazards regression analyses of variables associated with cancer-specific survival.

\begin{tabular}{|c|c|c|c|c|}
\hline \multirow[t]{2}{*}{ Characteristics } & \multicolumn{2}{|l|}{ Univariate analysis } & \multicolumn{2}{|l|}{ Multivariable analysis } \\
\hline & $\mathrm{HR}(95 \% \mathrm{Cl})$ & $P$ & $\mathrm{HR}(95 \% \mathrm{Cl})$ & $P$ \\
\hline Insured status & & 0.143 & $\mathrm{NI}$ & \\
\hline uninsured/unknown & Reference & & & \\
\hline any medicaid & $1.331(0.703-2.521)$ & 0.379 & & \\
\hline insured & $0.773(0.530-1.127)$ & 0.181 & & \\
\hline Age & & $<0.001$ & & $\hat{0}_{0.001}$ \\
\hline$<40$ & Reference & & Reference & \\
\hline $40-65$ & $1.951(1.081-3.520)$ & 0.026 & $1.375(0.756-2.502)$ & 0.297 \\
\hline$\nabla 65$ & $3.833(2.072-7.091)$ & $<.001$ & $2.971(1.577,5.597)$ & 0.001 \\
\hline Gender & & & & 0.582 \\
\hline male & Reference & & Reference & \\
\hline female & $0.496(0.351-0.699)$ & $<.001$ & $1.110(0.766-1.608)$ & \\
\hline Marital status & & 0.074 & & 0.118 \\
\hline unmarried & Reference & & Reference & \\
\hline married & $0.728(0.514-1.031)$ & & $0.750(0.524-1.075)$ & \\
\hline Race & & 0.339 & $\mathrm{NI}$ & \\
\hline white & Reference & & & \\
\hline black & $1.004(0.554-1.819)$ & 0.991 & & \\
\hline other & $0.512(0.209-1.252)$ & 0.142 & & \\
\hline AJCC stage & & $\begin{array}{l}<.001 \\
0\end{array}$ & & $\hat{0}_{0.001}$ \\
\hline stage I & Reference & & Reference & \\
\hline stage II & $5.560(1.181,26.188)$ & 0.030 & $5.693(1.205-26.909)$ & 0.028 \\
\hline stage III & $7.880(1.529-40.615)$ & 0.014 & $10.474(1.978-55.472)$ & 0.006 \\
\hline stage IV & $\begin{array}{l}62.233(15.381- \\
251.805)\end{array}$ & $<.001$ & $\begin{array}{l}42.797(10.006- \\
183.040)\end{array}$ & $\begin{array}{l}< \\
0.001\end{array}$ \\
\hline
\end{tabular}




\begin{tabular}{|c|c|c|c|c|}
\hline \multirow[t]{2}{*}{ Characteristics } & \multicolumn{2}{|l|}{ Univariate analysis } & \multicolumn{2}{|l|}{ Multivariable analysis } \\
\hline & $\mathrm{HR}(95 \% \mathrm{Cl})$ & $P$ & $\mathrm{HR}(95 \% \mathrm{Cl})$ & $P$ \\
\hline Tumor size & & $<.001$ & & 0.931 \\
\hline$\varangle 2 \mathrm{~cm}$ & Reference & & Reference & \\
\hline $2-4 \mathrm{~cm}$ & $0.968(0.638-1.468)$ & 0.878 & $1.062(0.659-1.711)$ & 0.805 \\
\hline$\nabla 4 \mathrm{~cm}$ & $2.200(1.467-3.300)$ & $\begin{array}{l}<.001 \\
0\end{array}$ & $1.087(0.699-1.691)$ & 0.712 \\
\hline Solitary/multifocal & & 0.259 & $\mathrm{NI}$ & \\
\hline solitary tumor & Reference & & & \\
\hline multifocal tumor & $1.268(0.839-1.917)$ & & & \\
\hline Surgery & & $\stackrel{<}{0.001}$ & & $\begin{array}{l}< \\
0.001\end{array}$ \\
\hline no surgery & Reference & & Reference & \\
\hline lobectomy & $0.046(0.018-0.115)$ & ${ }_{0.001}^{<}$ & $0.286(0.107-0.764)$ & 0.013 \\
\hline $\begin{array}{l}\text { total/subtotal } \\
\text { thyroidectomy }\end{array}$ & $0.057(0.040,0.082)$ & $\begin{array}{l}<.001 \\
0.00\end{array}$ & $0.244(0.125-0.476)$ & $<0.001$ \\
\hline Lymph node dissection & & ${ }_{0.001}^{<}$ & & 0.039 \\
\hline none or biopsy & Reference & & Reference & \\
\hline $\begin{array}{l}1 \text { to } 3 \text { regional lymph } \\
\text { nodes }\end{array}$ & $0.536(0.305-0.942)$ & 0.030 & $1.443(0.740-2.810)$ & 0.282 \\
\hline$\geq 4$ regional lymph nodes & $0.456(0.317-0.654)$ & ${ }_{0.001}^{<}$ & $0.652(0.348-1.221)$ & 0.181 \\
\hline Chemotherapy & & $<_{0.001}^{<}$ & & 0.069 \\
\hline no/unknown & Reference & & Reference & \\
\hline yes & $11.254(7.723-16.400)$ & & $1.530(0.968-2.420)$ & \\
\hline Radiotherapy & & ${ }_{0.001}^{<}$ & & 0.001 \\
\hline no/unknown & Reference & & Reference & \\
\hline yes & $5.536(3.936-7.785)$ & & $1.820(1.270-2.608)$ & \\
\hline
\end{tabular}

Nomogram construction and validation 
Those significant independent risk factors predicted by multivariate analysis were used to construct the prognosis nomogram for predicting the CSS rates at 3 and 5 years among the adult MTC cases (Fig. 3). All the prognostic variables were rated according to the point scale on top of every nomogram, and the scores were added for the variables on the bottom of every nomogram to predict the CSS rates at 3 and 5 years. As suggested by the CSS prediction model based on the nomogram, the AJCC stage of tumor contributed the most to patient prognosis, whereas surgery, age, LND and radiotherapy ranked the second to fifth place in succession. Furthermore, calibration and C-index were adopted for the nomogram internal validation.For the CSS predicted by the nomogram, the value of C-index was 0.902 ( $95 \% \mathrm{Cl}: 0.878$ 0.926), which indicated that the nomogram-based model was accurate in prediction. Moreover, the calibration curves also implied that the survival predicted by the nomogram were highly consistent with the real survival (Fig. 4).

\section{Discussion}

To our knowledge, our research aimed to identify prognostic indicators in adult MTC patients based on the large population-based database. In total, 1279 adult MTC patients from the SEER database were analyzed. The SEER database renders assessment on large cross-section studies in large-scale cancer patients, and provides long-term follow-up without intrinsic institutional biases such as surgical expertise, selection, therapeutic preferences or referral ${ }^{15}$.

Tumor stage at diagnosis was the most significant independent clinical factor for survival. However, Roman et al believed that age was the most significant prognostic factor ${ }^{6}$.The reasons for the inconsistency are likely to be that we used the 8th AJCC stage and included only adult patients, while Roman et al used SEER summary stage and enrolled many pediatric patients. Nevertheless, we revealed that age was also an independent risk factor for prognosis ${ }^{5,16}$.

Unlike differentiated thyroid cancer, radioactive iodine is not concentrated in MTC cells, which are insensitive manipulation of thyroid-stimulating hormone. Despite the lately approved targeted therapies providing extensive therapeutic choices for patients with metastatic or progressive disease, surgical resection is still the first-line curative therapy in early-stage patients ${ }^{17}$. In particular, the extent of surgical resection and lymph node dissection are independent prognostic factors, which have been substantiated in various population-based clinical researches ${ }^{5,6,17,18}$. Traditional chemotherapy offers only limited benefit in MTC patients ${ }^{19}$. In addition, we found that radiotherapy did not bring survival benefits to MTC patients, but shortened the survival period, although Fersht et al had been reported that radiotherapy can partially alleviate MTC and improve the quality of life of patients ${ }^{20}$.

Nomogram-based 3- and 5-year CSS prediction in MTC was successfully constructed, which harbored favorable discrimination and calibrations indicated by internal validations. Nomogram, a user-friendly statistical method, can provide survival estimation in a specific subject only via simple graphical presentation ${ }^{21}$. Additionally, nomogram has been confirmed to harbor better prediction capacity over the 
conventional AJCC TNM staging systems in certain types of malignant tumors, recognized as an alternative or even a novel standard ${ }^{22,23}$.

There are several strengths in this study. The detailed clinicopathological information of MTC patients collected from the SEER database ensured that our constructed nomogram was accurate. In addition, the adopted clinicopathological indicators were easily available, making it convenient for the clinical application of the nomogram. At the same time, there were certain limitations in our research. Firstly, the nomogram was established by retrospectively collecting data from the SEER database, possibly resulting in selection bias. Secondly, several clinicopathological factors correlated with prognosis were not available in the SEER database, including serum calcitonin level and RET mutations situation, which are main focus in our future study. Thirdly, the nomogram might be more convincing in the case of external validation by another independent large-scale sample.

\section{Conclusion}

For the first time, we constructed and validated a nomogram, which was capable of providing personalized estimates of 3-and 5-year CSS rates in adult MTC patients in this large population-based study. It could help clinicians to better understand the disease and identify patients at high risk. Nevertheless, the further exploration of unknown prognostic indicators is of great significant for the optimization of nomogram, and external validation is also warranted.

\section{Declarations}

\section{Funding}

No funding.

\section{Conflict of interest}

All authors declare that they have no conflicts of interest.

\section{Author contributions}

Xu-he Zhang conceived the study. Xiao Li searched the database and literature. Xiao Li, Yu-jia Lin, Xianzhi Wang, Xiang-min Zeng and Qiang-Li discussed and analyzed the data. Xiao Li wrote the manuscript. Xu-he Zhang revised the manuscript. All authors approved the final version.

\section{References}

1 Sherman, S. I. Thyroid carcinoma. Lancet (London, England)361, 501-511, doi:10.1016/s01406736(03)12488-9 (2003).

2 Thomas, C. M., Asa, S. L., Ezzat, S., Sawka, A. M. \& Goldstein, D. Diagnosis and pathologic characteristics of medullary thyroid carcinoma-review of current guidelines. Current oncology (Toronto, 
Ont.)26, 338-344, doi:10.3747/co.26.5539 (2019).

3 Starenki, D. \& Park, J. I. Pediatric Medullary Thyroid Carcinoma. Journal of pediatric oncology3, 29-37, doi:10.14205/2309-3021.2015.03.02.1 (2015).

4 Meng, K., Luo, H., Chen, H., Guo, H. \& Xia, W. Prognostic value of numbers of metastatic lymph node in medullary thyroid carcinoma: A population-based study using the SEER 18 database. Medicine98, e13884, doi:10.1097/md.0000000000013884 (2019).

5 Kebebew, E., Ituarte, P. H., Siperstein, A. E., Duh, Q. Y. \& Clark, O. H. Medullary thyroid carcinoma: clinical characteristics, treatment, prognostic factors, and a comparison of staging systems. Cancer88, 11391148, doi:10.1002/(sici)1097-0142(20000301)88:5<1139::aid-cncr26>3.0.co;2-z (2000).

6 Roman, S., Lin, R. \& Sosa, J. A. Prognosis of medullary thyroid carcinoma: demographic, clinical, and pathologic predictors of survival in 1252 cases. Cancer107, 2134-2142, doi:10.1002/cncr.22244 (2006).

7 lasonos, A., Schrag, D., Raj, G. V. \& Panageas, K. S. How to build and interpret a nomogram for cancer prognosis. Journal of clinical oncology : official journal of the American Society of Clinical Oncology26, 1364-1370, doi:10.1200/jco.2007.12.9791 (2008).

8 Sternberg, C. N. Are nomograms better than currently available stage groupings for bladder cancer? Journal of clinical oncology : official journal of the American Society of Clinical Oncology24, 3819-3820, doi:10.1200/jco.2006.07.1290 (2006).

9 Fang, C. et al. Nomogram individually predicts the overall survival of patients with gastroenteropancreatic neuroendocrine neoplasms. British journal of cancer117, 1544-1550, doi:10.1038/bjc.2017.315 (2017).

10 Tian, T. et al. Nomogram construction for predicting survival of patients with non-small cell lung cancer with malignant pleural or pericardial effusion based on SEER analysis of 10,268 patients. Oncology letters19, 449-459, doi:10.3892/ol.2019.11112 (2020).

11 Dai, D., Jin, H. \& Wang, X. Nomogram for predicting survival in triple-negative breast cancer patients with histology of infiltrating duct carcinoma: a population-based study. American journal of cancer research8, 1576-1585 (2018).

12 Guan, Y. J., Fang, S. Y., Chen, L. L. \& Li, Z. D. Development and validation of prognostic nomograms for medullary thyroid cancer. OncoTargets and therapy12, 2299-2309, doi:10.2147/ott.s196205 (2019).

13 Kim, C. Y., Collier, C. D., Liu, R. W. \& Getty, P. J. Are Limb-sparing Surgical Resections Comparable to Amputation for Patients With Pelvic Chondrosarcoma? A Case-control, Propensity Score-matched Analysis of the National Cancer Database. Clinical orthopaedics and related research477, 596-605, doi:10.1097/corr.0000000000000622 (2019). 
14 Liang, W. et al. Development and validation of a nomogram for predicting survival in patients with resected non-small-cell lung cancer. Journal of clinical oncology : official journal of the American Society of Clinical Oncology33, 861-869, doi:10.1200/jco.2014.56.6661 (2015).

15 Huang, Q. et al. Prognostic factors and clinical outcomes in adult primary gliosarcoma patients: a Surveillance, Epidemiology, and End Results (SEER) analysis from 2004 to 2015. British journal of neurosurgery, 1-7, doi:10.1080/02688697.2019.1699903 (2019).

16 Hundahl, S. A., Fleming, I. D., Fremgen, A. M. \& Menck, H. R. A National Cancer Data Base report on 53,856 cases of thyroid carcinoma treated in the U.S., 1985-1995 [see commetns]. Cancer83, 2638-2648, doi:10.1002/(sici)1097-0142(19981215)83:12<2638::aid-cncr31>3.0.co;2-1 (1998).

17 Roy, M., Chen, H. \& Sippel, R. S. Current understanding and management of medullary thyroid cancer. The oncologist18, 1093-1100, doi:10.1634/theoncologist.2013-0053 (2013).

18 Jin, L. X. \& Moley, J. F. Surgery for lymph node metastases of medullary thyroid carcinoma: A review. Cancer122, 358-366, doi:10.1002/cncr.29761 (2016).

19 Kloos, R. T. et al. Medullary thyroid cancer: management guidelines of the American Thyroid Association. Thyroid : official journal of the American Thyroid Association19, 565-612, doi:10.1089/thy.2008.0403 (2009).

20 Fersht, N., Vini, L., A'Hern, R. \& Harmer, C. The role of radiotherapy in the management of elevated calcitonin after surgery for medullary thyroid cancer. Thyroid : official journal of the American Thyroid Association11, 1161-1168, doi:10.1089/10507250152741019 (2001).

21 Balachandran, V. P., Gonen, M., Smith, J. J. \& DeMatteo, R. P. Nomograms in oncology: more than meets the eye. The Lancet. Oncology16, e173-180, doi:10.1016/s1470-2045(14)71116-7 (2015).

22 Cao, J. et al. Clinical Nomogram for Predicting Survival of Esophageal Cancer Patients after Esophagectomy. Scientific reports6, 26684, doi:10.1038/srep26684 (2016).

$23 \mathrm{Sheu}, \mathrm{T}$. et al. Nomogram for predicting symptom severity during radiation therapy for head and neck cancer. Otolaryngology-head and neck surgery : official journal of American Academy of OtolaryngologyHead and Neck Surgery151, 619-626, doi:10.1177/0194599814545746 (2014).

\section{Figures}




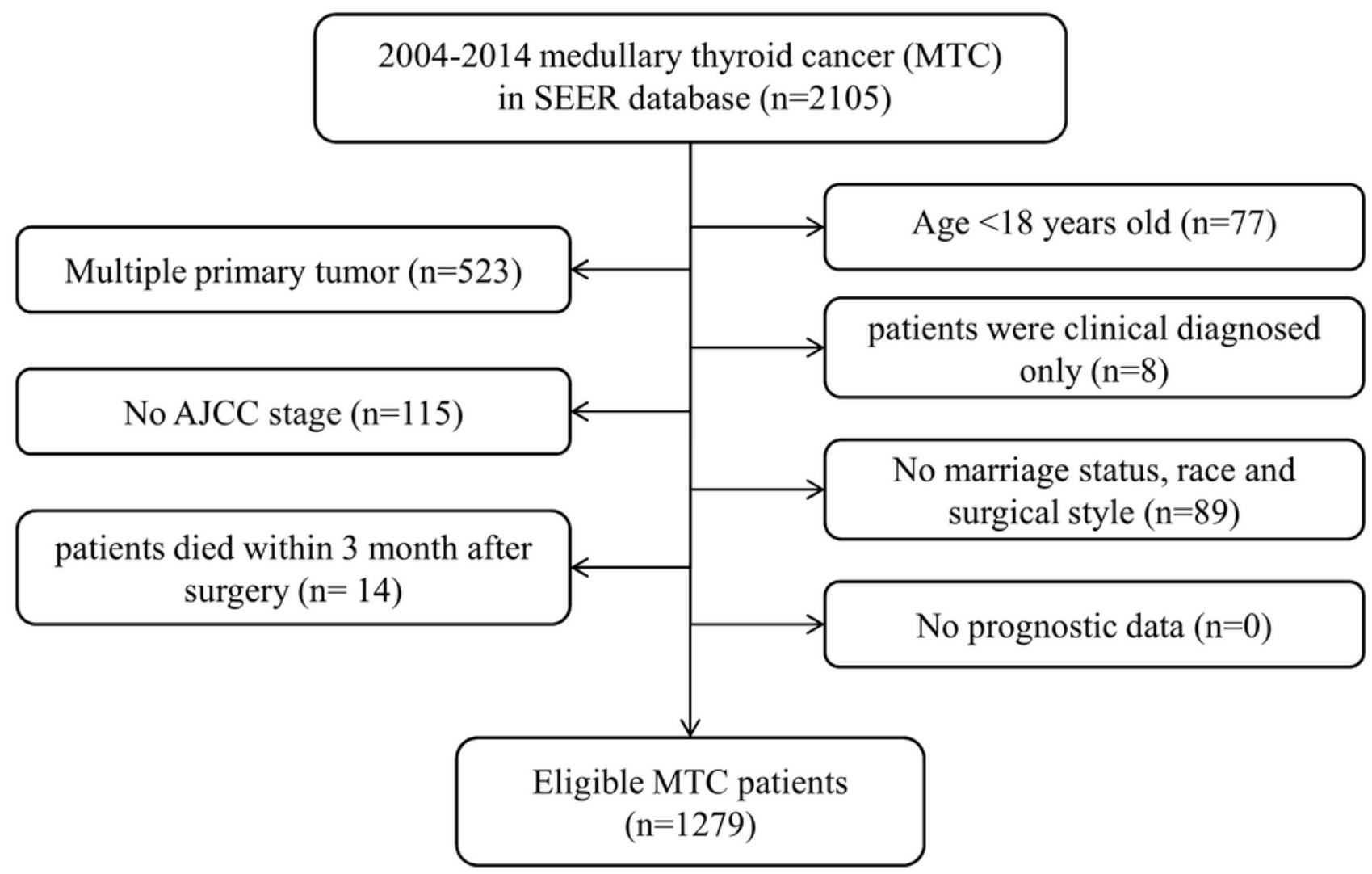

Figure 1

Flow chart for screening eligible patients. 


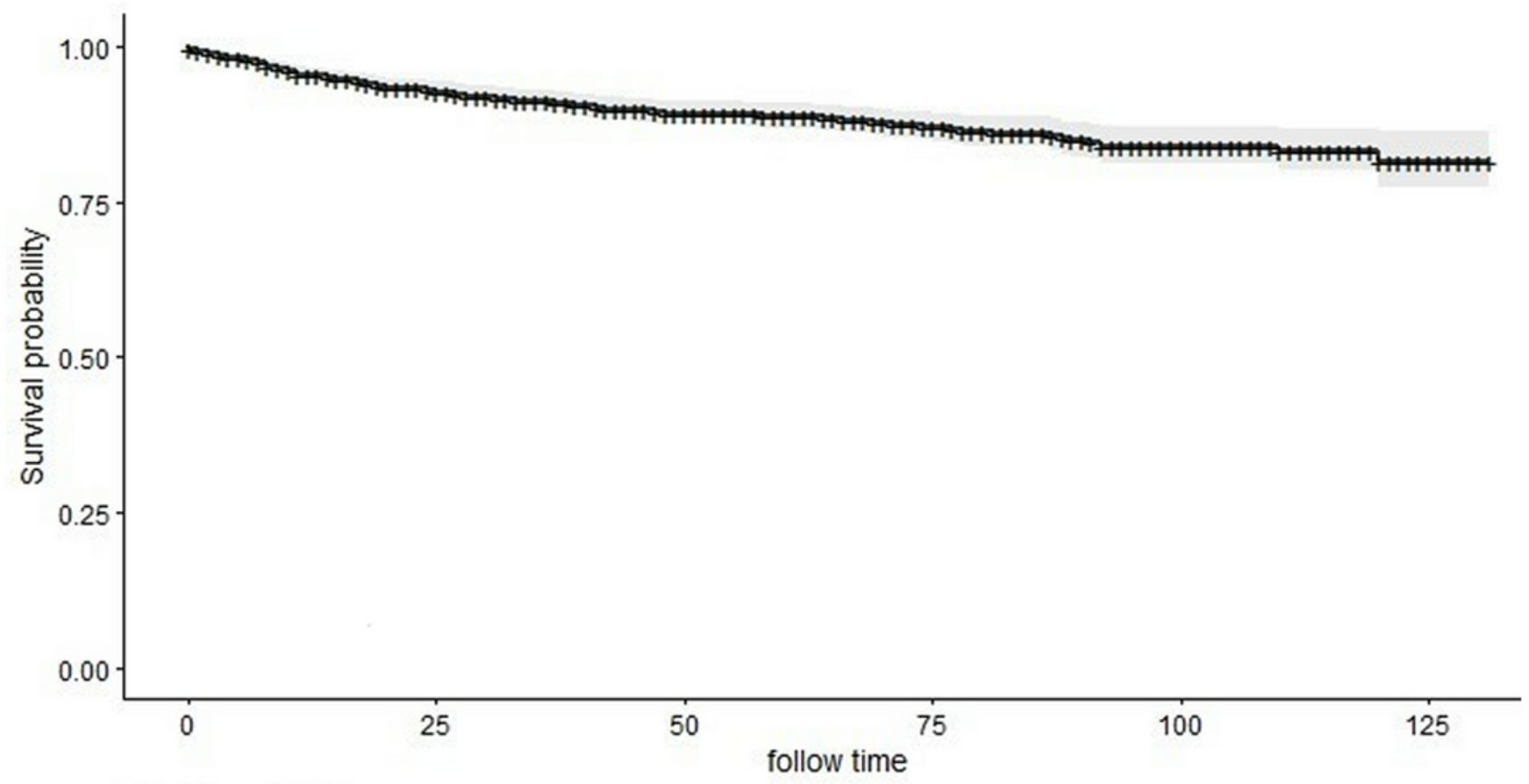

Number at risk

\begin{tabular}{|c|c|c|c|c|c|c|}
\hline All & 1279 & 883 & 605 & 364 & 182 & 31 \\
\hline & 0 & 25 & 50 & 75 & 100 & 125 \\
\hline
\end{tabular}

Figure 2

Kaplan-Meier curves for cancer-specific survival (CSS) of included patients. 
Points

$\begin{array}{lllllllllll}0 & 10 & 20 & 30 & 40 & 50 & 60 & 70 & 80 & 90 & 100\end{array}$

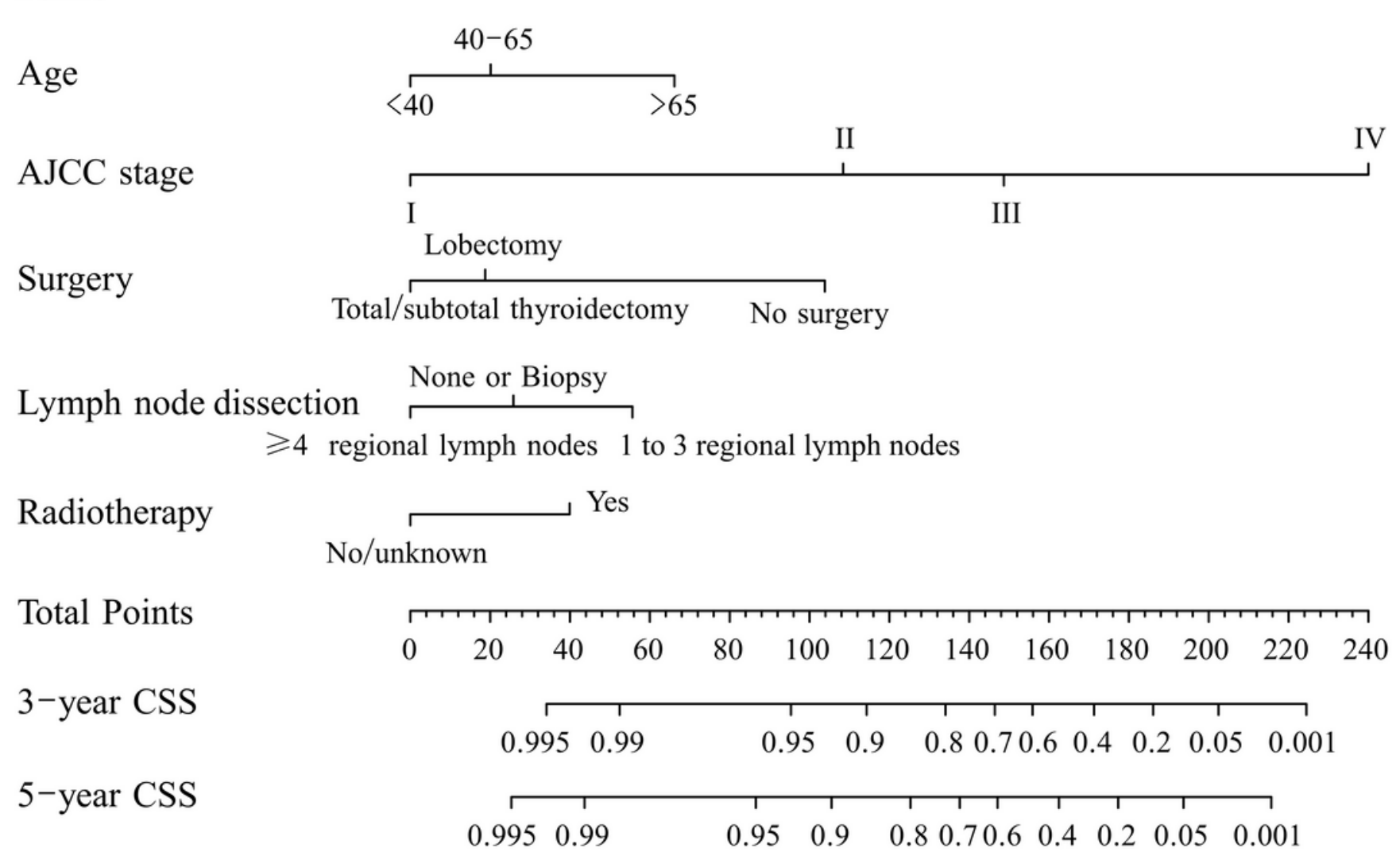

\section{Figure 3}

The nomogram for predicting 3- and 5-year cancer-specific survival (CSS) of patients with medullary thyroid carcinoma (MTC). 
A

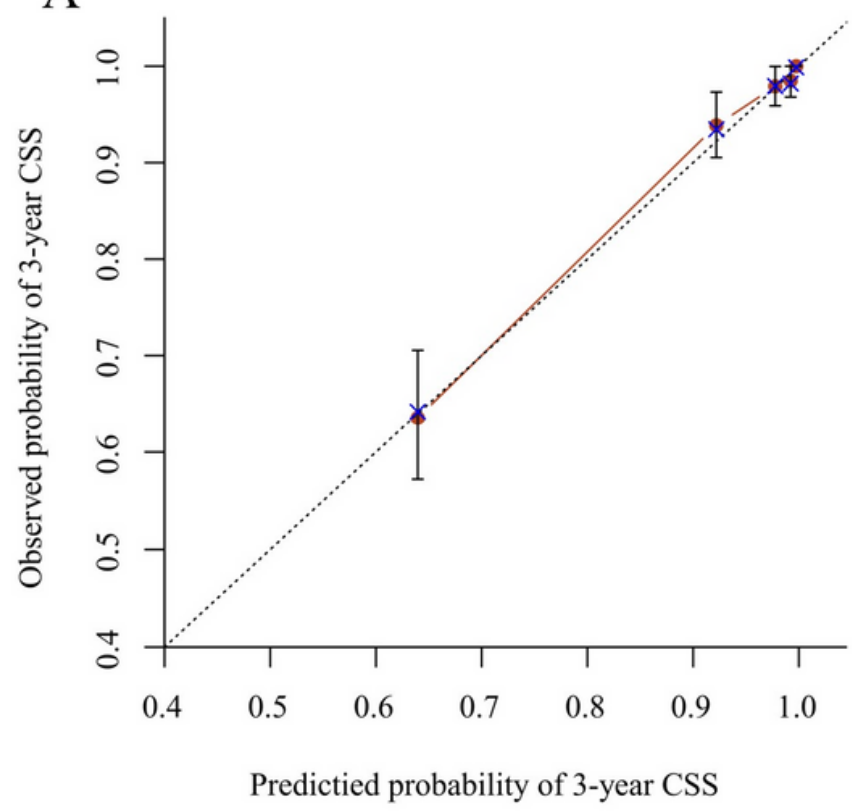

B

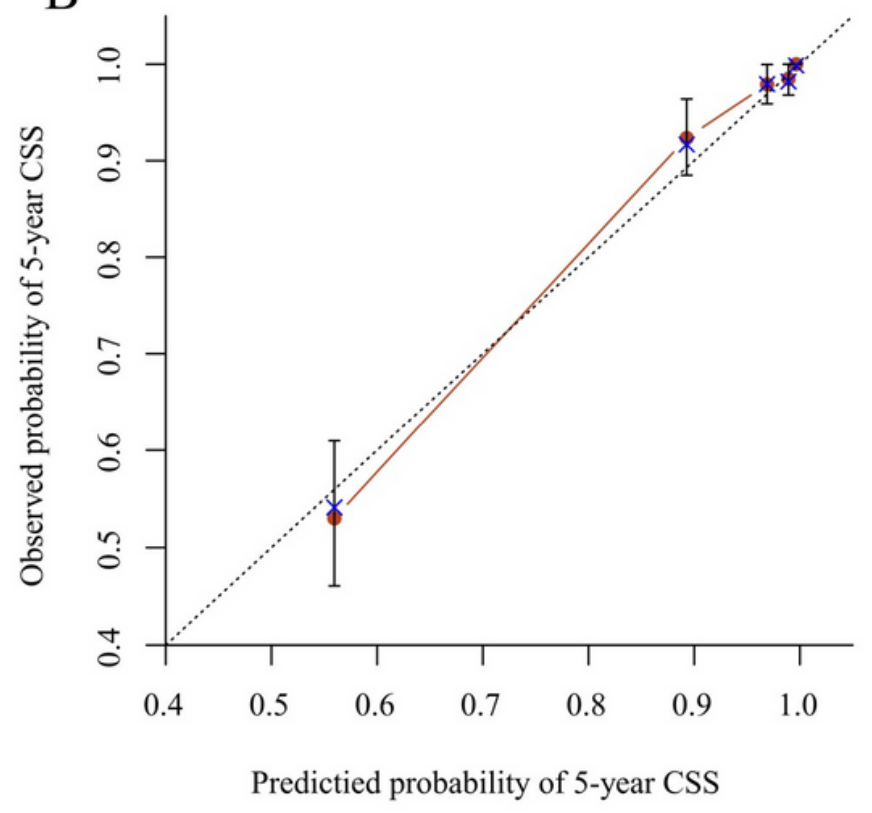

\section{Figure 4}

Calibration plots of the nomogram for 3-year (A) and 5-year (B) cancer-specific survival (CSS) prediction in the training set. The $\mathrm{X}$-axis represents the nomogram-predicted probability of survival; the $\mathrm{Y}$-axis represents the actual CSS probability. Plots along the 45-degree line indicate a perfect calibration model in which the predicted probabilities are identical to the actual outcomes. Vertical bars indicate $95 \%$ confidence intervals. 